\title{
Effective field theories with instantons
}

\section{Citation}

Georgi, Howard, and Samuel T Osofsky. 1994. "Effective Field Theories with Instantons." Nuclear Physics B 420 (1-2): 94-117. https://doi.org/10.1016/0550-3213(94)90376-x.

\section{Permanent link}

http://nrs.harvard.edu/urn-3:HUL.InstRepos:41467452

\section{Terms of Use}

This article was downloaded from Harvard University's DASH repository, and is made available under the terms and conditions applicable to Other Posted Material, as set forth at http:// nrs.harvard.edu/urn-3:HUL.InstRepos:dash.current.terms-of-use\#LAA

\section{Share Your Story}

The Harvard community has made this article openly available.

Please share how this access benefits you. Submit a story.

Accessibility 
HUTP-93/A021

\title{
Effective Field Theories with Instantons*
}

\author{
Howard Georgi*† and Samuel T. Osofsky*† \\ Lyman Laboratory of Physics \\ Harvard University \\ Cambridge, MA 02138
}

We explain how nonperturbative effects can be systematically included when constructing an effective field theory. We concentrate in particular on the matching calculation, by which a low energy theory without a heavy particle degree of freedom is matched onto the full theory. The matching calculation requires some new formalism, because for a large class of theories, instantons occur in a continuous range of scales from far below the mass of the heavy particle to far above. We show how the complete IR finiteness of the usual matching formalism arises in this new context. As an example, we formally integrate out a heavy quark in QCD.

$07 / 93$

\footnotetext{
* Research supported in part by the National Science Foundation, under grant \#PHY-9218167, and in part by the Texas National Research Laboratory Commission under grant \#RGFY93-278B.

* Research supported in part by the National Science Foundation, under grant \#PHY-9218167, and in part by the Texas National Research Laboratory Commission under grant \#RGFY93-278B.

$\dagger$ (georgi@huhepl.harvard.edu)

*†† (osofsky@huhepl.harvard.edu)
} 


\section{Introduction}

In constructing an effective field theory ${ }^{1}$ from a more fundamental theory, one starts with the renormalization scale $\mu$ at some high scale and then uses the renormalization group to evolve $\mu$ continuously down to lower and lower scales, eventually encountering the mass scale $M$ of one of the particles in the theory. At scales below $M$, perturbative arguments imply decoupling: in a physical renormalization scheme, graphs with virtual heavy particles are suppressed by powers of $p / M$, where $p$ is an external momentum in the low energy theory [2].

However, for tractable evaluation of higher loop diagrams, and in order to simplify the renormalization group equations, a mass independent scheme such as MS is more attractive than a physical scheme. In a mass independent scheme, decoupling must be included by hand: after $\mu$ has been evolved to $M$, the theory is matched onto a low energy theory where the only degrees of freedom are the light fields. The matching is achieved order by order in some small parameter, such as in a loop expansion. The new theory is then evolved via the renormalization group to lower scales.

In this paper we investigate how instanton effects can be included in this picture, in particular in the matching calculation, since we find that the continuous part of the renormalization group is only trivially modified. There are two very different reasons for doing this.

In a large class of theories, instantons occur in a continuous range of scales from far below $M$ to far above. By this we mean that the functional integral is dominated by solutions (or approximate solutions) of the bosonic Euclidean equations of motion, and the bosonic action is classically scale invariant over that range. However, the effective field theory is necessarily an expansion to some finite order $n$ in $p / m$, where $p$ is a momentum in the low energy theory. For fixed $n$ the effective theory will duplicate the physics of the full theory (to some accuracy) when $p / m<1$, but its behavior will be wildly different when $p>m$. This leads to the first motivation for studying nonperturbative contributions to matching: to determine the role of instantons, and in particular the role of instantons smaller than $1 / m$, in the low energy theory. These small instantons necessarily involve momentum scales $p$ with $p>m$. We will show that, as might be expected, the small instantons can very naturally be included in the matching corrections, and the large ones left in the effective theory. To do the matching calculation we will find it useful to think of the dilute gas

1 For a review of effective field theory, see [1]. 
approximation as a map from Lagrangians to Lagrangians, and to simultaneously expand in instanton density and the number of loops.

The second reason is that matching should provide one of the few calculations involving instantons (in a classically scale invariant, asymptotically free theory) which does not suffer from IR problems. These problems arise for the same reason perturbative calculations at small momenta fail in such theories, although in this case it is large instantons that create the problem: for low energy scales the coupling grows too large for perturbation theory, or a semiclassical expansion, to be trusted. But a matching calculation should have no IR problems, because all the IR physics should cancel. In particular, the dilute gas approximation, by which we will mean a perturbative expansion in instanton density at the heavy fermion scale, will be trustworthy, if instantons at that scale and above are dilute. This is in contrast to most instanton calculations, where large instantons ruin the calculation whatever scale it is performed at. We will see this IR cancellation in a specific example.

The remainder of the paper is organized as follows. In sect. 2, we review effective field theories. In sect. 3 we briefly review instantons and comment on renormalization and the renormalization group in that context. In sect. 4 we explain the matching calculation. In sect. 5 we formally apply the method of sect. 4 to matching at a heavy quark threshold in QCD. Finally, in sect. 6 we comment about practical calculations.

\section{Effective Field Theories}

In this section, we briefly review the formalism of effective field theories, following [1].

Given a quantum field theory (whether effective or not), a low energy effective field theory can be constructed as follows. Begin at a very large scale, that is, with the renormalization scale, $\mu$, very large. In a strongly interacting theory or a theory with unknown physics at high energy, this starting scale should be sufficiently large that nonrenormalizable interactions produced at higher scales are too small to be relevant. In a renormalizable, weakly interacting theory, $\mu$ should be above the masses of all the particles, where the effective theory is given simply by the renormalizable theory, with no nonrenormalizable terms.

In this region, the physics is described by a set of fields, $\Phi$, describing the heaviest particles, of mass $M$, and a set of light particle fields, $\phi$, describing all the lighter particles. The Lagrangian has the form

$$
\mathcal{L}_{H}(\Phi, \phi)+\mathcal{L}_{L}(\phi)
$$


where $\mathcal{L}_{L}(\phi)$ contains all the terms that depend only on the light fields, and $\mathcal{L}_{H}(\Phi, \phi)$ is everything else. The theory is then evolved down to lower scales. As long as no particle masses are encountered, this evolution is described by the renormalization group. However, when $\mu$ drops below the mass, $M$, of the heavy particles, decoupling should be put in by hand by switching to an effective theory without the heavy particles. In the process, the parameters of the theory change, and new interactions, some nonrenormalizable, may be introduced. The Lagrangian of the effective theory below $M$ has the form

$$
\mathcal{L}_{L}(\phi)+\delta \mathcal{L}(\phi)
$$

where $\delta \mathcal{L}(\phi)$ is the "matching correction" that contains the new interactions.

Both the changes in existing parameters, and the coefficients of the new interactions are computed by "matching" the physics just below the boundary in the two theories. Matching determines the sizes of the nonrenormalizable terms associated with the heavy particles. Generally, the only way we have of calculating matching corrections is in perturbation theory in some small parameter. It can be done in a loop expansion which, if there are no factors of $\hbar$ hidden in the parameters of the Lagrangian, is equivalent to an expansion in powers of $\hbar$,

$$
\delta \mathcal{L}=\sum_{l=0}^{\infty} \delta \mathcal{L}^{(l)} .
$$

The matching correction, $\delta \mathcal{L}(\phi)$, should be chosen so that the physics of the $\phi$ particles is the same in the two theories at $\mu=M$, just at the boundary.

The strongest form of this equivalence that we might impose is that all one-lightparticle irreducible (1LPI) functions with external light particles are the same. By 1LPI is meant a graph that cannot be disconnected by cutting a single light particle line. We can describe this in terms of the equality of the light particle effective actions in the two descriptions. The light particle effective action in the low energy theory is just the effective action. In the high energy theory, the light particle effective action is defined as follows: construct the generating functional for connected Feynman graphs with light particles external lines, and then Legendre transform it. This gives the generating functional for 1LPI graphs. Then the matching condition is ${ }^{2}$

$$
S\left[\mathcal{L}_{H}+\mathcal{L}_{L}\right]=S\left[\mathcal{L}_{L}+\delta \mathcal{L}\right]
$$

2 A weaker condition could be imposed - that the $S$-matrix for light particle scattering is the same in the two theories. There are arguments (which we do not understand) that in some cases only this weaker form of equivalence can be imposed: attempting to match the greens functions, rather then just the $S$-matrix, leads to a nonlocal effective field theory [3]. 
We have changed the notation in (2.4) from that of [1] in two ways for typographical convenience. We have suppressed the dependence on the classical field, $\phi$, and we have indicated the dependence on the Lagrangian by square brackets.

Formally, we imagine expanding $S[\mathcal{L}]$, where $\mathcal{L}$ is an arbitrary Lagrangian, in the number of loops $l$ :

$$
S[\mathcal{L}]=\sum_{l} S^{(l)}[\mathcal{L}]
$$

It should be stressed that $S^{(l)}$ is a projection of $S$ onto the l-loop part. If there are terms from loop effects already present in $\mathcal{L}$, then the loop-order of these terms must be taken into account when calculating $S^{(l)}$.

At tree level, the light particle effective action in the low energy theory is just the Lagrangian itself, thus

$$
S^{(0)}\left[\mathcal{L}_{L}+\delta \mathcal{L}^{(0)}\right]=\int \mathcal{L}_{L}+\int \delta \mathcal{L}^{(0)}
$$

In the high energy theory, the light particle effective action is the sum of all 1LPI tree graphs. Thus it is a sum of the light particle Lagrangian plus all trees with external light particles and only internal heavy particle lines:

$$
S^{(0)}\left[\mathcal{L}_{H}+\mathcal{L}_{L}\right]=\int \mathcal{L}_{L}+\int\left\{\begin{array}{l}
\text { virtual heavy } \\
\text { particle trees }
\end{array}\right\}
$$

Thus

$$
\begin{aligned}
\int \delta \mathcal{L}^{(0)} & =S^{(0)}\left[\mathcal{L}_{H}+\mathcal{L}_{L}\right]-S^{(0)}\left[\mathcal{L}_{L}\right] \\
& =\int\left\{\begin{array}{l}
\text { virtual heavy } \\
\text { particle trees }
\end{array}\right\} .
\end{aligned}
$$

This is rather trivial, but it is already clear that the matching correction is a difference between a calculation in the full theory and one in the low energy effective theory.

We must sharpen the definition of the "change in the Lagrangian", $\delta \mathcal{L}$ (for the moment we consider this object to an arbitrary number of loops), in that up to this point it is nonlocal, because, for example, of its dependence on $p / M$ through the virtual heavy particle propagators (here $p$ refers to any of the external momenta). However, it will turn out to be analytic in $p / M$ everywhere in the region relevant to the low energy theory. Thus it can be expanded in powers with the higher order terms steadily decreasing in importance. It can then be dealt with, to any finite order in the momentum expansion, as a local Lagrangian. We will always interpret the induced operators as if $\delta \mathcal{L}$ where 
expanded to some finite order - that is, as a local Lagrangian. In Feynman diagrams we will indicate the operators to be momentum expanded by surrounding them by a dotted line; an example is shown in fig. 1, where thick lines represent a heavy particle and thin lines a light one. It should be stressed that the object in fig. 1 indicated by a dotted line is a point-like, local operator.

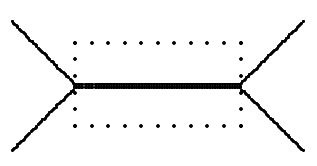

Figure 1: The low energy approximation to a heavy particle propagator (thick line) is indicated by surrounding the propagator by a dotted line.

Evaluating $\delta \mathcal{L}$ is more interesting at one loop. From the general properties of the effective action, we can write

$$
S^{(1)}\left[\mathcal{L}_{L}+\delta \mathcal{L}\right]=S^{(1)}\left[\mathcal{L}_{L}+\delta \mathcal{L}^{(0)}\right]+\int \delta \mathcal{L}^{(1)}
$$

This is because the one loop term, $\delta \mathcal{L}^{(1)}$, can appear only as a tree diagram. A loop graph with an inclusion of $\delta \mathcal{L}^{(1)}$ would give a contribution even higher order in $\hbar$. Thus the matching condition to one loop can be written:

$$
\int \delta \mathcal{L}^{(1)}=S^{(1)}\left[\mathcal{L}_{H}+\mathcal{L}_{L}\right]-S^{(1)}\left[\mathcal{L}_{L}+\delta \mathcal{L}^{(0)}\right]
$$

In this equation, both $S^{(1)}\left[\mathcal{L}_{H}+\mathcal{L}_{L}\right]$ and $S^{(1)}\left[\mathcal{L}_{L}+\delta \mathcal{L}^{(0)}\right]$ are nonlocal functions of the fields in which the nonlocality is determined by the long distance physics. If there are massless particles in the theory, the 1PI functions may not even be analytic in the momenta, $p$, as $p \rightarrow 0$. However, in the difference, all the long distance physics that gives rise to this nonanalyticity cancels. All the remaining nonlocality is due to the propagation of the heavy particles and the result can be expanded in powers of $p / M$ to give a sum of local operators.

The matching calculation is IR finite. An infrared divergence always arises from a loop integration over small momenta. But for small momentum, the full theory and the effective theory give the same physics, by construction. If we kept an infinite number of terms of the momentum expansion in the effective theory, the physics would be exactly the same and there would be no contribution at all from the loop integral for momenta smaller 
than $M$. In practice, to compute the one loop matching corrections to some order in the momentum expansion, it is only necessary to include enough terms to eliminate all the IR divergences in the loop integral for the matching correction. If there is still an IR divergence at one loop to some order in the momentum expansion, it simply means that more terms in the momentum expansion must be included in the tree level matching corrections. This argument works to each order in the loop expansion. Matching calculations are always completely IR finite.

We will see that all of the essential features of perturbative matching as described in this section continue to hold when we include the effects of instantons.

\section{Instantons}

If the functional integral for a relativistic quantum field theory is defined as an analytic continuation from that of a Euclidean theory, then solutions and approximate solutions to the Euclidean equations of motion are important configurations in the integral. Expanding semiclassically about these configurations should in some regime be a good approximation to the functional integral. That is all we will say here about this vast subject.

From now on, when we refer to a quantum field theory, we refer to the Euclidean version.

\subsection{Collective Coordinates}

We briefly review the collective coordinate formalism [4]. Let $S[\Phi]$ be the local classical action for the bosonic sector of some quantum field theory, with $\Phi$ being the bosonic fields. Suppose also that $S$ has some set of continuous global symmetries. If $\Phi_{c l}$ is a solution to the equations of motion, it will break some of these symmetries, and therefore must be a member of a family of solutions $\Phi_{c l}(a)$, where $a$ represents one parameter for each broken global symmetry degree of freedom. We will call these parameters collective coordinates.

Expand the quantum field as $\Phi=\Phi_{c l}(a)+g \eta$, with $\eta$ the quantum fluctuation, and $g$ the theory's small coupling. Then the action can be expanded in $\eta$ :

$$
S[\Phi]=S\left[\Phi_{c l}(a)\right]+\frac{g^{2}}{2} \eta \cdot\left(\left.\frac{\delta^{2} S}{\delta \Phi^{2}}\right|_{\Phi_{c l}(a)}\right) \cdot \eta+\cdots
$$

Here the dot represents an integral over space-time and a summation over symmetry/flavor indices. 
Define the local operator $\mathcal{O}(a)$ by

$$
\frac{g^{2}}{2} \eta \cdot\left(\left.\frac{\delta^{2} S}{\delta \Phi^{2}}\right|_{\Phi_{c l}(a)}\right) \cdot \eta=\frac{1}{2} \int d^{4} x \eta \mathcal{O}(a, x) \eta
$$

Let $\zeta_{n}(a)$ be the orthonormal modes of $\mathcal{O}(a)$ :

$$
\begin{aligned}
\mathcal{O}(a) \zeta_{n}(a) & =\lambda_{n}(a) \zeta_{n}(a) \\
\zeta_{m}(a) \cdot \zeta_{n}(a) & =\delta_{m n}
\end{aligned}
$$

and expand $\eta=\sum_{k} \alpha_{k} \zeta_{k}(a)$. The functional integral can be written:

$$
\begin{aligned}
Z & =\int\left(\prod_{k} d \alpha_{k}\right) \mathrm{e}^{-S[\Phi]} \\
& =\mathrm{e}^{-S\left[\Phi_{c l}\right]}\left(\prod_{k} d \alpha_{k}\right) \mathrm{e}^{-1 / 2 \eta \cdot \mathcal{O} \eta}
\end{aligned}
$$

For each $a$-parameter there will be a zero mode of $\mathcal{O}(a)$ :

$$
\zeta_{k}^{(0)}(a)=N_{k} \frac{\partial \Phi_{c l}(a)}{\partial a_{k}}
$$

where $N_{k}$ is defined by

$$
\begin{aligned}
1 & =\zeta_{k}^{(0)}(a) \cdot \zeta_{k}^{(0)}(a) \\
& =N_{k}^{2} \frac{\partial \Phi_{c l}(a)}{\partial a_{k}} \cdot \frac{\partial \Phi_{c l}(a)}{\partial a_{k}}
\end{aligned}
$$

Then $N_{k}=\left(\frac{\partial \Phi_{c l}(a)}{\partial a_{k}} \cdot \frac{\partial \Phi_{c l}(a)}{\partial a_{k}}\right)^{-1 / 2}$.

As in the Fadeev-Popov method, for each normalizable zero mode ${ }^{3}$ insert

$$
1=\int d a_{k} \delta\left(\eta \cdot \zeta_{k}^{(0)}(a)\right) \frac{\partial}{\partial a_{k}}\left(\eta \cdot \zeta_{k}^{(0)}(a)\right)
$$

The $\delta$-function just restricts the fluctuation to be orthogonal to the zero mode, while

$$
\begin{aligned}
\frac{\partial}{\partial a_{k}}\left(\eta \cdot \zeta_{k}^{(0)}(a)\right) & =\left[\frac{\partial}{\partial a_{k}}\left(\frac{\Phi-\Phi_{c l}(a)}{g}\right)\right] \cdot \zeta_{k}^{(0)}(a)+\eta \cdot \frac{\partial \zeta_{k}^{(0)}(a)}{\partial a_{k}} \\
& =-\frac{1}{g N_{k}(a)}\left(1-Q_{k}\right)
\end{aligned}
$$

3 Only the normalizable zero modes make the functional determinant zero [5]. 
where

$$
Q_{k}=g N_{k}(a) \eta \cdot \frac{\partial \zeta_{k}^{(0)}(a)}{\partial a_{k}}
$$

That is, ignoring the overall sign,

$$
1=\frac{1}{g}\left(\int d a_{k} \delta\left(\eta \cdot \zeta_{k}^{(0)}(a)\right)\left(\frac{\partial \Phi_{c l}(a)}{\partial a_{k}} \cdot \frac{\partial \Phi_{c l}(a)}{\partial a_{k}}\right)^{1 / 2}\right)\left(1-Q_{k}\right)
$$

We rederived the well-known collective coordinate result primarily to emphasize that the usual formalism can be thought of as a zeroeth order approximation in $g$ : a factor of $Q_{k}$ comes with one power of $g$, and usually none is kept. But since we will be doing a loop expansion, we must keep all interactions generated by $Q_{k}$. However, when the matching calculation is performed to any finite order, these interactions will give rise to only a finite number of diagrams for each term induced in the low energy theory.

In any case it is no surprise that collective coordinates, just like semi-classical or perturbative calculations, are not very meaningful if the coupling is large.

\subsection{Quasi-Collective Coordinates}

In the previous section, we considered collective-coordinates arising from exact global symmetries. If we consider the Euclidean action as a height function over the space of field configurations, these symmetries appear as exactly level troughs, along the bottom of which lie the families of exact solutions. With a collective coordinate, we can perform the integral along the bottom of the trough exactly. We mean to treat families of approximate solutions in a similar way.

For example, suppose $\Phi_{c l}(a)$ and $\Phi_{c l}\left(a^{\prime}\right)$ are instantons, and $a$ and $a^{\prime}$ are chosen so that the instanton centers $z, z^{\prime}$ are widely separated. Since typically $\Phi_{c l}$ is localized in space-time, it must be that $\Phi\left(a, a^{\prime}\right)=\Phi_{c l}(a)+\Phi_{c l}\left(a^{\prime}\right)$ is a solution to the equations of motion up to terms which vanish as $\left|z-z^{\prime}\right| / \rho$ tends to infinity for fixed instanton size $\rho$.

Alternatively, suppose the action is classically scale invariant. Any localized nonsingular instanton solution must have a characteristic size and so break this symmetry. Then there must be a collective-coordinate $\mu_{I}$ corresponding to the (inverse-) size of the instanton.

If the scale invariance is explicitly broken by adding a $\Phi$-mass $m$ to the action, then some aspects of this picture must still hold at scales much higher than $m$, even though simple scaling arguments imply there are no exact instanton solutions [6][7]. This is because 
at high enough scales, the mass term should be a small perturbation. So we expect approximate instantons similar (at distances smaller than $m^{-1}$ ) to those present when $m=0$; they will still be naturally characterized by a scale parameter $\mu_{I}$, with $\mu_{I}>>m$.

If we again consider the Euclidean action as a height function over the space of field configurations, both of these examples appear as nearly level troughs, along the bottom of which lie families of approximate solutions parameterized in the first case by $z, z^{\prime}$ and in the second by $\mu_{I}$. For $\left|z-z^{\prime}\right| / \rho$ and $\mu_{I} / m>>1$ these troughs become asymptotically flat and should be treated as are the true collective coordinate troughs to well approximate the functional integral. Two ways to do this are the "constrained instanton" method [6] and the "valley" method [8].

In either of these methods, a parameter is introduced for integrating along the approximately level troughs. The methods differ essentially only in that for the valley method the path of this integration always passes through precisely the lowest point along a slice of field configurations orthogonal to the trough direction, while the constrained instanton path passes near, but not necessarily through, that point. Since each point of the integration path represents a classical field configuration (which is then being expanded about) the definitions of the trough parameters will not be precisely the same, though both methods should give the same answer for any calculation in a region where the troughs are nearly flat. Since there is no need here to distinguish between these (or any other similar) methods, we will refer to these trough parameters as quasi-collective coordinates, and to the classical field configurations as quasi-instantons, without specifying precisely what this means.

Note that in the first example, the quasi-instanton would be $\Phi\left(a, a^{\prime}\right)=\Phi_{c l}(a)+$ $\Phi_{c l}\left(a^{\prime}\right)+\cdots$ where the unwritten terms are definite, but method dependent functions of $a, a^{\prime}$. In the second example the quasi-instanton indexed by $\mu_{I}$ is approximately the same as the instanton of size $\mu_{I}^{-1}$ from its center out to a radius $r \sim m^{-1}$, after which it falls off as $\mathrm{e}^{-m r}[6]$.

In the course of doing the quasi-collective coordinate integral, regions of parameter space will be encountered where quasi-instantons come closer together than their sizes, and where $\mu_{I} \sim m$. Here the troughs are no longer even approximately flat. In the first example will always imagine integrating through these trouble spots anyway, and in the second integrating $\mu_{I}$ from some method dependent scale, of order $m$, up to infinity. This cavalier approach is justified as follows: for the first example, we will only be considering situations where the dilute gas approximation is valid, so that the functional integral is 
dominated by field configurations that look like a collection of widely separated instantons. If the regions where instanton overlap are important, than this approximation fails. For the second example, we will only be considering in detail situations where the configurations with $\mu_{i}>>m$ are the important ones; more specifically there will be a heavy particle mass $M>>m$ and configurations with $\mu_{I}<<M$ will be unimportant. We will show that this is the situation at a heavy quark threshold in QCD, for example, where the instantons occur in a massless field and $m=0$. In section 4.1, we will discuss a case where this condition is not met when the (quasi-) instantons occur in the heavy field.

Expanding about a quasi-instanton leaves the formalism of the previous section completely unchanged, except for the effects of expanding about a different field configuration. For example, the expansion in equation (3.1) will now have a term linear in the quantum fluctuation. For this reason we will typically drop the distinction between instanton and quasi-instanton, and between collective coordinate and quasi-collective coordinate, for the rest of this paper.

\subsection{Perturbative Expansion About an Instanton}

Perturbing around an instanton, the generator of $\eta$-correlators is:

$$
Z[J]=\left.\mathrm{e}^{-S_{c l}} \int(\mathcal{D} \eta)\right|_{a} \exp \int\left[\mathcal{O}^{(1)}(a) \eta-\eta \mathcal{O}(a) \eta+\mathcal{O}^{(3)}(a) \eta^{3}+\cdots+J \eta\right]
$$

where $\left\{\mathcal{O}^{(n)}(a)\right\}(n>2)$ are from the higher order terms in equation $(3.1)$ (and $\mathcal{O}^{(1)}(a)$ is present only in the case of a quasi-instanton).

Define the instanton density:

$$
D(a)=\mathrm{e}^{-S_{c l}} \prod_{k} \frac{1}{g}\left(\frac{\partial \Phi_{c l}(a)}{\partial a_{k}} \cdot \frac{\partial \Phi_{c l}(a)}{\partial a_{k}}\right)^{1 / 2}
$$

(where the product is over all zero (or quasi-zero) modes). Then switching to collective coordinates gives

$$
\begin{aligned}
Z[J]= & \left.\int d a D(a) \int(\mathcal{D} \eta)^{\prime}\right|_{a} \prod_{k}\left(1-Q_{k}\right) \\
& \exp \int\left[\mathcal{O}^{(1)}(a) \eta-\eta \mathcal{O}(a) \eta+\mathcal{O}^{(3)}(a) \eta^{3}+\cdots+J \eta\right]
\end{aligned}
$$

where $Q_{k}$ are defined in equation (3.9) and the prime in $\left.\int(\mathcal{D} \eta)^{\prime}\right|_{a}$ denotes integration orthogonal to the zero modes. 
Doing the constrained $\eta$-integral is essentially the same problem as fixing a gauge in standard gauge theory, and we can proceed with a simplified version of a method often used there [9]. Instead of equation (3.7) use

$$
1=\int d a_{k} \delta\left(\eta \cdot \zeta_{k}^{(0)}(a)-C_{k}\right) \frac{\partial}{\partial a_{k}}\left(\eta \cdot \zeta_{k}^{(0)}(a)\right)
$$

The rest of the collective coordinate formalism is not modified, except that now $\eta \cdot \zeta_{k}^{(0)}(a)$ is restricted to be an arbitrary constant $C_{k}$ rather than zero.

Inserting this form of 1 into the functional integral yields a result independent of $C_{k}$, so we can also insert $\int d C_{k} \mathrm{e}^{-C_{k}^{2} / \alpha_{k}^{\prime}}$ where $\alpha_{k}^{\prime}$ is an arbitrary constant which, like the gauge fixing parameter $\alpha$, must cancel out at the end of any calculation of a physical quantity. This insertion changes the functional integral by an irrelevant overall constant. Therefore

$$
\begin{aligned}
Z[J]= & \left.\int d a D(a) \int(\mathcal{D} \eta)\right|_{a} \prod_{k}\left(1-Q_{k}\right) \\
& \exp \int\left[\mathcal{O}^{(1)}(a) \eta-\eta \widetilde{\mathcal{O}}(a) \eta+\mathcal{O}^{(3)}(a) \eta^{3}+\cdots+J \eta\right]
\end{aligned}
$$

where

$$
\eta \cdot \widetilde{\mathcal{O}}(a) \cdot \eta=\eta \cdot \mathcal{O}(a) \cdot \eta+\int d^{4} x \sum\left(\eta \cdot \zeta_{k}^{(0)}\right)^{2} / \alpha_{k}^{\prime}
$$

For the moment it is most convenient to use the free propagator $\mathcal{O}_{0}$, treating the rest of the terms as interactions, because otherwise we aren't certain how to use the formalism of the second section. (However, this will be remedied in section 3.7.) So define $\mathcal{O}_{1}^{(2)}(a)=\widetilde{\mathcal{O}}(a)-\mathcal{O}_{0}$. It will also be convenient to similarly separate the higher order interactions into those depending on the instanton and those which don't (these last are $\eta$-self interactions that were present in the original Lagrangian): $\mathcal{O}^{(n)}(a)=\mathcal{O}_{0}^{(n)}+\mathcal{O}_{1}^{(n)}(a)$. Then

$$
\begin{aligned}
& Z[J]=\left.\int d a D(a) \int(\mathcal{D} \eta)\right|_{a} \prod_{k}\left(1-Q_{k}\right) \\
& \quad \exp \int\left[-\eta \mathcal{O}_{0} \eta+\mathcal{O}_{0}^{(3)} \eta^{3}+\cdots+\mathcal{O}_{1}^{(1)}(a) \eta+\mathcal{O}_{1}^{(2)}(a) \eta^{2}+\cdots+J \eta\right]
\end{aligned}
$$

In fig. 2 we introduce a Feynman notation for the theory. 


$$
\begin{aligned}
& \longrightarrow=\eta \text {-propagator } \quad \bullet \quad=\int d a D(a) \\
& \bar{\ldots}=\mathcal{O}_{0}^{(n)} \quad \widehat{\nwarrow}=\int d a D(a) \mathcal{O}_{1}^{(n)}(a) \\
& (n \eta \text {-legs; } n>2) \quad(n \eta \text {-legs } ; n>0)
\end{aligned}
$$

Figure 2: Feynman notation.

\subsection{Dilute Gas Approximation}

The basic assumption of the dilute gas approximation (DGA) is that the functional integral is dominated by field configurations that look like a collection of widely separated instantons. Corrections can be added order by order in instanton density $D$.

In the $n$-instanton sector the action can be expanded as

$$
\begin{aligned}
S\left[\sum_{I=1}^{n} \Phi_{c l}\left(a_{I}\right)+\eta\right]=n S_{c l}+\int\left[-\eta \mathcal{O}_{0} \eta+\mathcal{O}_{0}^{(3)} \eta^{3}+\cdots\right] \\
+\sum_{I} \int\left[\mathcal{O}_{1}^{(1)}\left(a_{I}\right) \eta+\cdots\right] \\
+\sum_{k=2}^{n} \int(k-\text { body instanton }- \text { instanton interactions })
\end{aligned}
$$

The sum over instanton-instanton interactions is defined so as to make the equation correct; a $k$-body term consists of a function of $k$ instanton coordinates coupled to any number (including zero) of $\eta$ 's. In principle the sum could be computed, but we will see that it contributes at next to leading order in $D$. The $\left\{\mathcal{O}_{1}^{(n)}\right\}$ are just the 1-body interactions written out more explicitly.

The generator of $\eta$-correlators is now

$$
\begin{aligned}
Z_{\mathrm{DGA}}[J] & =\sum_{n=0}^{\infty} \frac{1}{n !}\left(\prod_{I=1}^{n} \int d a_{I} D\left(a_{I}\right)\right) \int \mathcal{D} \eta \prod_{I=1}^{n}\left[\prod_{k}\left(1-Q_{k}\left(a_{I}\right)\right)\right] \\
& \exp \left\{\int\left[-\eta \mathcal{O}_{0} \eta+\mathcal{O}_{0}^{(3)} \eta^{3}+\cdots\right]+\sum_{I=1}^{n} \int\left[\mathcal{O}^{(1)}\left(a_{I}\right) \eta+\cdots\right]+\int J \eta\right. \\
& \left.+\sum_{k=2}^{n} \int(k-\text { body instanton - instanton interactions })\right\}
\end{aligned}
$$


(The factor of $n$ ! is necessary to avoid double counting identical instantons.) Here the sum over $k$-body interactions, which has the same basic form as before, accounts not only for the errors in expanding the action, but also in the collective coordinate procedure. Since we have assumed the same zero modes etc. as if the instantons were infinitely separated, this procedure requires corrections that look like instanton-instanton interactions etc. Again, the sum could in principle be computed, but will contribute at next to leading order in $D$.

Absorb the factors of $1-Q_{k}$ into the interactions by using $1-Q=\mathrm{e}^{\ln (1-Q)}$; this amounts to setting

$$
\mathcal{O}_{1}^{(n)}(a) \rightarrow \mathcal{O}_{1}^{(n)}(a)-\frac{1}{n} \sum_{k} Q_{k}^{n}(a)
$$

It follows that

$$
\begin{aligned}
Z_{\mathrm{DGA}}[J]=\int \mathcal{D} \eta \exp \left\{\int\left[-\eta \mathcal{O}_{0} \eta+\mathcal{O}_{0}^{(3)} \eta^{3}+\cdots+J \eta\right]+\right. \\
\left.\int d a D(a) \mathrm{e} \int\left[\mathcal{O}_{1}^{(1)}(a) \eta+\mathcal{O}_{1}^{(2)}(a) \eta^{2}+\cdots\right]+\mathcal{O}\left(D^{2}\right)\right\}
\end{aligned}
$$

We are dealing explicitly with only leading order in $D$, but it should be stressed that we can always calculate to higher order.

Now, in addition to the diagrams shown in fig. 2, there are interactions at $\mathcal{O}\left(D^{1}\right)$ obtained from products of 1-body operators; an example is shown in fig. 3 .

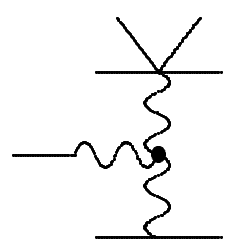

Figure 3: Diagram for the interaction $\int d a D(a)\left[\mathcal{O}_{1}^{(1)}(a) \eta\right]\left[\mathcal{O}_{1}^{(4)}(a) \eta^{4}\right]\left[\mathcal{O}_{1}^{(2)}(a) \eta^{2}\right]$

\subsection{DGA as a map from Lagrangians to Lagrangians}

It will be convenient to think of the DGA as a map $F$ from Lagrangians to Lagrangians. That is, if $\mathcal{L}$ is the Lagrangian before including instanton effects, so that the generator of $\Phi$-correlators is

$$
Z[J]=\int \mathcal{D} \Phi \mathrm{e}^{\int(-\mathcal{L}+J \Phi)}
$$

then the DGA generator of $\eta$-correlators can be written as

$$
Z_{\mathrm{DGA}}[J]=\int \mathcal{D} \eta \mathrm{e}^{\int(-F[\mathcal{L}]+J \eta)}
$$


where the functional integral is to be performed perturbatively in the trivial instanton sector. From (3.21) it follows that

$$
\int F[\mathcal{L}]=\int \mathcal{L}-\int d a D(a) \mathrm{e}^{\int\left[\mathcal{O}_{1}^{(1)}(a) \eta+\cdots\right]}+\mathcal{O}\left(D^{2}\right)
$$

The term "Lagrangian" has been used loosely here as in $(2.8)$ and $(2.10)$. If $\mathcal{L}$ is a local Lagrangian, then generally $F[\mathcal{L}]$ will be nonlocal because of the nonlocality of the instanton induced interactions. However we will see that in a matching calculation this nonlocality is completely under control, in that the matching corrections, when calculated to any finite order in the momentum expansion, will be local. But any instantons remaining in the effective theory will still generally give nonlocal interactions.

\subsection{Renormalization and Renormalization Group}

Wherever we write the instanton density $D$ or coupling $g$ we mean the renormalized instanton density $D^{(\mu)}$ and coupling $g(\mu)$, where $\mu$ is the renormalization group scale. $D^{(\mu)}$ is defined by (3.12), where any couplings occurring in the right hand side are the renormalized ones. As explained in the introduction, a mass independent scheme is desirable so we imagine choosing the counterterms via MS.

\subsection{Nonperturbative interactions vs propagating in an instanton background}

There is no small coupling associated with an instanton interaction except for the instanton density. A diagram where the same instanton interacts several times with a given quantum particle line (with no other instanton interactions) is still $\mathcal{O}\left(D^{1}\right)$. Therefore, whenever we are considering a diagram with one or more instantons, we must imagine summing up all diagrams where each instanton interacts with each quantum line any number of times. A notation for this is indicated in fig. 4.

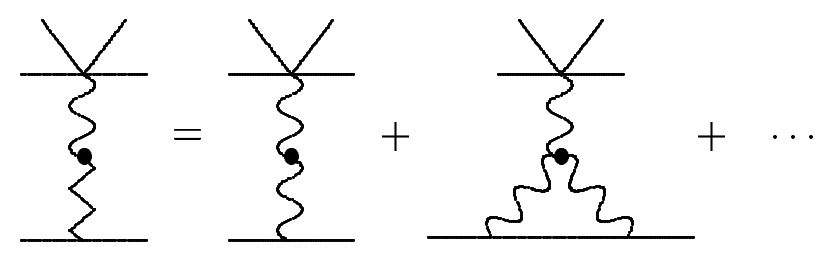

Figure 4: The jagged line indicates the sum on the right. 
Actually, the sum indicated by the jagged line should be thought of as the particle propagator in an instanton background (before integrating over the instanton's collective coordinates), minus the free propagator, rather than as a series of nonperturbative interactions.

However, when an instanton connects two different quantum lines, or interacts with the same line both before and after another instanton does, the diagram should be thought of as having a nonperturbative interaction. For the purposes of counting loops etc, these interactions can be shrunk to a point. An example is shown in fig. 5 .

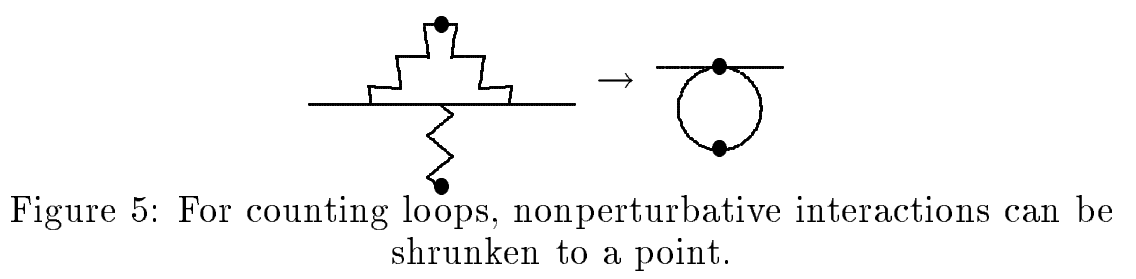

\section{Matching in Effective Field Theories With Instantons}

As pointed out in [1], there are two cases to consider.

\subsection{Instantons in the Heavy Field}

Let $\Phi$ represent now just the light fields, and suppose $\Psi$ is a heavy bosonic field, of mass $M$, with instantons. As explained in section 3.2, the instantons will be indexed by a quasi-collective coordinate $\mu_{I}$ (and have characteristic size $\mu_{I}^{-1}$ ) where $\mu_{I}$ runs from some scale above $M$ to infinity. Most of an instanton's Fourier transform will be in momenta higher than $\mu_{I}$, and in particular, higher than $M$. The low energy theory has no $\Psi$ and therefore no instantons; they will appear only through the matching corrections. The fact that the instanton is cutoff at frequencies below $M$ means that the momentum expansion in the low energy theory will be good.

The matching equation can be taken to be

$$
S_{\text {pert }}\left[F\left[\mathcal{L}_{H}+\mathcal{L}_{L}\right]\right]=S_{\text {pert }}\left[\mathcal{L}_{L}+\delta \mathcal{L}\right]
$$

where $S_{\text {pert }}$ is the 1LPI effective action, evaluated perturbatively about the trivial vacuum, and the renormalization scale is $\mu=M$. Note that $S_{\text {pert }}[\mathcal{L}]$ is defined to be the functional constructed via a loop expansion from the interactions present in $\mathcal{L}$, whether or not some of those interactions arise from instantons. In particular, suppose $\mathcal{L}$ is the Lagrangian for 
some theory with instantons (although no instanton effects on $\mathcal{L}$ have yet been included), and let $S[\mathcal{L}]$ be the theory's effective action. Then even assuming the validity of the loop expansion, we do not expect $S_{\text {pert }}[\mathcal{L}]=S[\mathcal{L}]$ since $S_{\text {pert }}$ ignores instanton effects; rather we expect (within the loop and dilute gas approximations) that $S_{\text {pert }}[F[\mathcal{L}]]=S[\mathcal{L}]$.

Because the instantons occur in the heavy field, the quasi-collective coordinate method may fail. This is because instantons with $\mu_{I}$-values ranging all the way down to their quasi-instanton-scheme dependent cutoff scale $\sim M$ may be important. If the calculation is scheme dependent, then a better method for dealing with the quasi-instantons is needed to calculate the matching corrections exactly. However, since all schemes should give roughly similar answers, the matching corrections can at least be estimated.

\subsection{Instantons in a Light Field}

Now suppose one of the light bosonic fields, $\phi$, has instantons, and let $\Psi$ be a heavy bosonic or fermionic field of mass $M$. This situation is more complicated because there will generally be instanton effects both in the full and effective theories. At scales far above the $\phi$-mass (or at any scale if $\phi$ is massless) the $\phi$-sector of the action will be approximately scale invariant, and therefore the instantons must come in families indexed by size. Then, as explained in the third section, there will be a collective coordinate $\mu_{I}$ corresponding to the instanton scale; $\mu_{I}$ must be integrated from some scale above the $\phi$-mass to infinity. For a given $\mu_{I}$ the instanton's Fourier transform will be cutoff by the $\phi$-mass in the IR and by $\mu_{I}$ in the UV.

If the $\phi$ is massless, as in QCD, then instanton calculations in the theory may suffer severe IR problems from the $\mu_{I} \rightarrow 0$ limit. Also, as explained in the introduction, small instantons $\left(\mu_{I}>M\right)$ would be problematic if present in the low energy theory because of their incompatibility with the low energy approximations to operators induced by $\Psi$.

The matching calculation is free of the first problem because of the cancellation of IR physics, and naturally avoids the second one. The low energy theory is still affected by instantons both with $\mu_{I}$ above and below $M$. But the small ones $\left(\mu_{I}>M\right)$ are present only in the matching corrections.

To realize this, it will be convenient to generalize the DGA map $F$ as follows: Let $F_{\Lambda}[\mathcal{L}]$ be the Lagrangian obtained by applying the DGA to $\mathcal{L}$, using the $\phi$-instantons, but only integrating $\mu_{I}$ up to the scale $\Lambda$. (If $\Lambda$ is below the scale where $\phi$-instantons exist, then $F_{\Lambda}[\mathcal{L}]=\mathcal{L}$.) 
The matching condition, applied at $\mu=M$, can be taken to be

$$
S_{\text {pert }}\left[F_{\infty}\left[\mathcal{L}_{H}+\mathcal{L}_{L}\right]\right]=S_{\text {pert }}\left[F_{M}\left[\mathcal{L}_{L}+\delta \mathcal{L}\right]\right]
$$

where $S_{\text {pert }}$ is the 1LPI effective action, evaluated perturbatively about the trivial vacuum. Any arbitrariness on the right hand side is just a definition of $\delta \mathcal{L}$.

To solve for $\delta \mathcal{L}$, expand in loops and renormalized instanton density $D^{(\mu)}$ (here $\mathcal{L}$ is an arbitrary Lagrangian):

$$
\begin{aligned}
\mathcal{L} & =\sum_{l, d} \mathcal{L}^{(l, d)} \\
S_{\text {pert }}[\mathcal{L}] & =\sum_{l, d} S_{\text {pert }}^{(l, d)}[\mathcal{L}] \\
\delta \mathcal{L} & =\sum_{l, d} \delta \mathcal{L}^{(l, d)} \\
F_{\Lambda}[\mathcal{L}] & =\sum_{l, d} F_{\Lambda}^{(l, d)}[\mathcal{L}]
\end{aligned}
$$

It should be stressed that $S_{\text {pert }}^{(l, d)}[\mathcal{L}]$ and $F_{\Lambda}^{(l, d)}[\mathcal{L}]$ are projections of $S_{\text {pert }}$ and $F_{\Lambda}$ onto the l-loop, $d$-instanton density part. If there are terms from loop effects or involving factors of instanton density already present in $\mathcal{L}$, then the $(l, d)$-order of these terms must be taken into account when calculating $S_{\text {pert }}^{(l, d)}[\mathcal{L}]$ and $F_{\Lambda}^{(l, d)}[\mathcal{L}]$.

It is now possible to solve for $\delta \mathcal{L}$ order by order in $(l, d)$. We define the following partial sums:

$$
\begin{aligned}
& G^{[l, d]}=\sum_{l^{\prime}=0}^{l} \sum_{d^{\prime}=0}^{d} G^{\left(l^{\prime}, d^{\prime}\right)} \\
& G^{[l, d)}=\sum_{l^{\prime}=0}^{l} G^{\left(l^{\prime}, d\right)} \\
& G^{(l, d]}=\sum_{d^{\prime}=0}^{d} G^{\left(l, d^{\prime}\right)}
\end{aligned}
$$

where $G$ is any of the objects $\mathcal{L}, \delta \mathcal{L}$, or $F_{\Lambda}$. As explained in the second section, by cluster arguments:

$$
S_{\text {pert }}^{(l, d)}\left[\mathcal{L}^{[l-1, d]}+\mathcal{L}^{(l, d]}\right]=S_{\text {pert }}^{(l, d)}\left[\mathcal{L}^{[l-1, d]}\right]+\int \mathcal{L}^{(l, d)}
$$

By counting powers of $D^{(\mu)}$, there is a similar rule for $F_{\Lambda}$ :

$$
F_{\Lambda}^{(l, d)}\left[\mathcal{L}^{[l, d-1]}+\mathcal{L}^{[l, d)}\right]=F_{\Lambda}^{(l, d)}\left[\mathcal{L}^{[l, d-1]}\right]+\mathcal{L}^{(l, d)}
$$


Note that (4.6) implies

$$
F_{\Lambda}^{(l, 0)}\left[\mathcal{L}^{(l, 0)}\right]=\mathcal{L}^{(l, 0)}
$$

With these rules, if we know $\delta \mathcal{L}$ to $\mathcal{O}(l-1, d)$ and to $\mathcal{O}(l, d-1)$, we can compute it to compute to $\mathcal{O}(l, d)$ as follows:

$$
\begin{array}{ll}
S_{\text {pert }}^{(l, d)} & {\left[F_{\infty}\left[\mathcal{L}_{H}+\mathcal{L}_{L}\right]\right]=S_{\text {pert }}\left[F_{M}\left[\mathcal{L}_{L}+\delta \mathcal{L}\right]\right]} \\
=S_{\text {pert }}^{[l, d]}\left[F_{M}^{[l, d]}\left[\mathcal{L}_{L}+\delta \mathcal{L}^{l l, d]}\right]\right] & \\
=S_{\text {pert }}^{(l, d)}\left[F_{M}^{[l, d]}\left[\mathcal{L}_{L}+\delta \mathcal{L}^{[l, d-1]}\right]+\delta \mathcal{L}^{[l, d)}\right] & \\
=S_{\text {pert }}^{(l, d)}\left[F_{M}^{[l-1, d]}\left[\mathcal{L}_{L}+\delta \mathcal{L}^{[l-1, d-1]}\right]+\delta \mathcal{L}^{[l-1, d)}\right. & \\
\left.\quad+F_{M}^{(l, d]}\left[\mathcal{L}_{L}+\delta \mathcal{L}^{[l, d-1]}\right]+\delta \mathcal{L}^{(l, d)}\right] & \\
=S_{\text {pert }}^{(l, d)}\left[F_{M}^{[l-1, d]}\left[\mathcal{L}_{L}+\delta \mathcal{L}^{[l-1, d-1]}\right]+\delta \mathcal{L}^{[l-1, d)}\right] & \\
\quad+\int\left[F_{M}^{(l, d)}\left[\mathcal{L}_{L}+\delta \mathcal{L}^{[l, d-1]}\right]+\delta \mathcal{L}^{(l, d)}\right] &
\end{array}
$$

Thus

$$
\begin{aligned}
\int \delta \mathcal{L}^{(l, d)} & =S_{\text {pert }}^{(l, d)}\left[F_{\infty}\left[\mathcal{L}_{H}+\mathcal{L}_{L}\right]\right] \\
& -S_{\text {pert }}^{(l, d)}\left[F_{M}^{[l-1, d]}\left[\mathcal{L}_{L}+\delta \mathcal{L}^{[l-1, d-1]}\right]+\delta \mathcal{L}^{[l-1, d)}\right] \\
& -\int F_{M}^{(l, d)}\left[\mathcal{L}_{L}+\delta \mathcal{L}^{[l, d-1]}\right]
\end{aligned}
$$

Equation (4.9) is the principle result of this paper. It is the generalization of the matching relations of [1] to include instanton-like effects. As expected it depends on $\delta \mathcal{L}$ up to $\mathcal{O}(l-1, d)$ and to $\mathcal{O}(l, d-1)$. Because it is still a matching correction, we also expect that the nonperturbative $\delta \mathcal{L}$ is IR finite and is nonlocal only on the heavy particle scale. We will show expicitly how this works in low orders in the examples discussed below.

\subsection{Anti-instantons}

We have been referring to all time-dependent non-trivial solutions of the Euclidean equations of motion as "instantons", but if the bosonic action is parity invariant then for any solution there will always be a parity reversed solution. If the two solutions are different then convention determines which is called the instanton and which the anti-instanton.

Precisely the same factors of instanton density are associated with a diagram containing anti-instanton interactions as would be were there only instantons, and for each leading order instanton-interaction there is precisely the same anti-instanton-interaction. 
Therefore the previous formalism is essentially unchanged. Instantons and anti-instantons are distinguishable from one another, however, so we introduce a Feynman notation in fig. 6 ; fig. 6 is to be compared with fig. 2 .

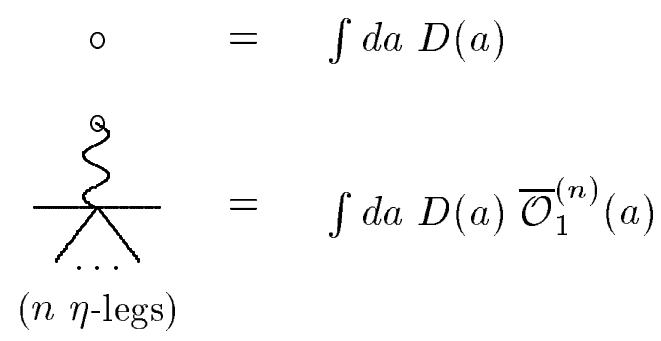

Figure 6: Feynman notation for anti-instantons.

Here $\overline{\mathcal{O}}_{1}^{(n)}(a)$ is the anti-instanton version of $\mathcal{O}_{1}^{(n)}(a)$ (see fig. 2).

\section{QCD}

As an example, we imagine matching out a heavy quark, mass $M$, in QCD, to order $(1,1)$. We assume that the DGA is good at the scale set by $M$ for instantons with $\mu_{I}>M$. After gauge fixing, the Lagrangian is

$$
\mathcal{L}=-\frac{1}{2 g^{2}} \operatorname{Tr} F^{2}(\mathcal{A})+\frac{1}{2 \alpha} \operatorname{Tr} G^{2}(\mathcal{A})+\sum_{F} \bar{\psi}_{F}\left(i \not D-m_{F}\right) \psi_{F}+\Phi^{\dagger} \frac{\delta G}{\delta \Omega} \Phi
$$

$F_{\mu \nu}$ and $D_{\mu}$ are, respectively, the field strength and covariant derivative for the gauge field $\mathcal{A}$, and $\left\{\psi_{F}\right\}$ are the fermions. The gauge group $\mathcal{G}$ is $S U(3)$ but we will act as if it were $S U(2)$ without loss of generality, since for a larger gauge group the instantons will live in the $S U(2)$ subgroups [10]. (From now on, we will suppress the fermion flavor index $F$.) $G[\mathcal{A}]$ is a gauge fixing functional, and $\Phi$ is the fermionic complex scalar Fadeev-Popov ghost; $\frac{\delta G}{\delta \Omega}$ is the variation of $G$ with respect to an infinitesimal gauge transformation. Finally, $g$ and $\alpha$ are, respectively, the gauge coupling and gauge fixing parameters.

In singular gauge the instanton is [11]

$$
A_{\mu}^{a}(x ; R, \mu, z)=\frac{R_{a b} \eta_{b \mu \nu}(x-z)_{\nu}}{(x-z)^{2}\left[1+\mu^{2}(x-z)^{2}\right]}
$$

where $R$ is an $S U(2)$ rotation matrix corresponding to the instanton's group orientation, $\mu$ is the instanton's inverse size, and $z$ its position. The $\eta$-symbol is defined in [7]; it maps 
antisymmetric tensors $T_{\mu \nu}$ in $S O(4)$, which is locally equivalent to $S O(3) \times S O(3)$, onto 3 -vectors in one of the $S O(3)$ groups. The anti-instanton $\bar{A}$ has the same expression but with $\eta \rightarrow \bar{\eta}$, where $\bar{\eta}$ maps into the other $S O(3)$.

We can define a multi-instanton configuration as

$$
\text { " } A_{n, \bar{n}} "=\sum_{i=1}^{n} A\left(z_{i}, R_{i}, \mu_{i}\right)+\sum_{i=1}^{\bar{n}} \bar{A}\left(z_{i}, R_{i}, \mu_{i}\right)
$$

We place " $A_{n, \bar{n}}$ " in quotes because we actually define $A_{n, \bar{n}}$ to be the above expression after a singular gauge transformation such that it is no longer singular.

\subsection{Background Field Gauge}

The expansion about an instanton necessitates partial use of the background field method [12][9]. We write $\mathcal{A}=A+g \eta$, where $A$ is a background classical field configuration and $\eta$ a quantum fluctuation.

Any physical quantity we calculate will be invariant under "quantum" gauge transformations:

$$
\begin{aligned}
& A \rightarrow A \\
& \eta \rightarrow \mathbf{g} \eta \mathbf{g}^{-1}+\mathbf{g} d \mathbf{g}^{-1}+\mathbf{g} A \mathbf{g}^{-1}-A
\end{aligned}
$$

(with $\mathbf{g}$ any $\mathcal{G}$-valued function). We can presumably always fix $\eta$, via a quantum gauge transformation, to background field gauge: $d \eta+[A, \eta]=0$

With this choice of gauge, physical quantities will be invariant under "background" gauge transformations:

$$
\begin{aligned}
& A \rightarrow \mathrm{g} A \mathrm{~g}^{-1}+\mathrm{g} d \mathrm{~g}^{-1} \\
& \eta \rightarrow \mathrm{g} \eta \mathbf{g}^{-1}
\end{aligned}
$$

Much of the utility of the background field method is that it calculates the effective action while avoiding calculating (and renormalizing) diagrams with external $\eta$-legs, but we won't take advantage of this feature, since the $\eta$-field will be kept as the gluon degree of freedom, and we are not concerned with maintaining explicit gauge invariance.

\subsection{Instanton Density}

Equations (3.12) and (5.2) give

$$
D(a) d a=(\text { const }) g^{-(\# \text { zero modes })} \mathrm{e}^{-8 \pi^{2} / g^{2}} d R \mu^{3} d \mu d^{4} z
$$

where $R, \mu, z$ are as in equation (5.2) and $d R$ is the group's invariant measure; both the number of zero modes and the overall constant depend on the gauge group $\mathcal{G}$. 


\subsection{Feynman Notation}

We adopt a notation essentially the same as before. The propogators are shown in fig. 7, and a subset of the interactions at $\mathcal{O}\left(D^{1}\right)$ are indicated in fig. 8.

$$
\begin{aligned}
& \text { lelel }=\eta \\
& \longrightarrow=\text { light fermion } \\
& \longrightarrow=\text { heavy fermion } \\
& --\rightarrow-- \text { ghost }
\end{aligned}
$$

Figure 7: Feynman notation for QCD propagators.

The complete set of interactions up to this order in $D$ involves all the usual QCD interactions, and all interactions obtained by connecting instantons in fig. 8 (an example of this was given in fig. 3). Note that there are interactions, coming from the collective coordinate terms, with an instanton connected to an arbitrary number of gluons.

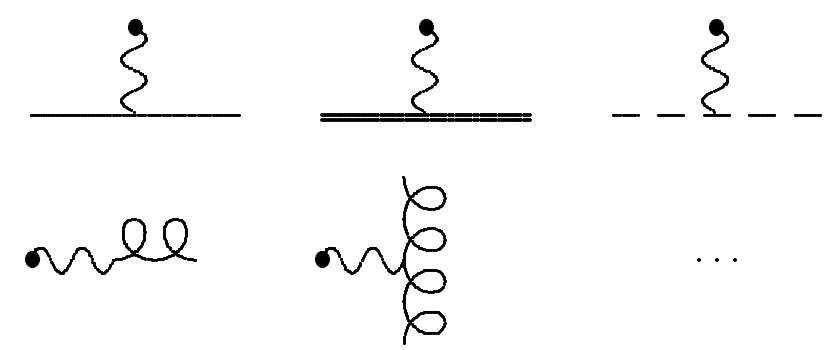

Figure 8: Subset of $\mathcal{O}\left(D^{1}\right)$ interactions.

For each instanton interaction, there is a corresponding anti-instanton interaction.

5.4. QCD Calculation to order $(0,0)$

To this order, equation (4.9) gives

$$
\int \delta \mathcal{L}^{(0,0)}=S_{\text {pert }}^{(0,0)}\left[F_{\infty}^{(0,0)}\left[\mathcal{L}_{H}+\mathcal{L}_{L}\right]\right]-\int F_{M}^{(0,0)}\left[\mathcal{L}_{L}\right]
$$

Using (4.7) and the fact that there are no virtual heavy particle trees in QCD, gives $\int \delta \mathcal{L}^{(0,0)}=0$. 
To this order, equation (4.9) gives

$$
\begin{aligned}
\int \delta \mathcal{L}^{(1,0)}= & S_{\mathrm{pert}}^{(1,0)}\left[F_{\infty}\left[\mathcal{L}_{H}+\mathcal{L}_{L}\right]\right]-S_{\mathrm{pert}}^{(1,0)}\left[F_{M}^{[0,0]}\left[\mathcal{L}_{L}\right]+\delta \mathcal{L}^{[0,0)}\right] \\
& -\int F_{M}^{(1,0)}\left[\mathcal{L}_{L}\right] \\
= & S_{\mathrm{pert}}^{(1,0)}\left[\mathcal{L}_{H}+\mathcal{L}_{L}\right]-S_{\mathrm{pert}}^{(1,0)}\left[\mathcal{L}_{L}\right]
\end{aligned}
$$

On the right hand side, all the diagrams cancel except the heavy fermion loops. That is, $\int \delta \mathcal{L}^{(1,0)}$ is the sum over (the low energy approximations to) heavy fermion loops, with gluon insertions (see fig. 9).

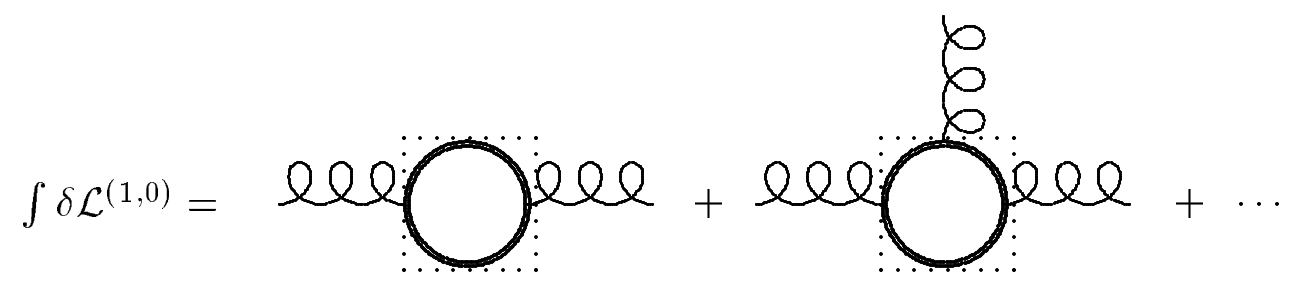

Figure 9: Matching contribution at $(1,0)$.

5.6. QCD Calculation to order $(0,1)$

To this order, equation (4.9) gives

$$
\begin{aligned}
\int \delta \mathcal{L}^{(0,1)} & =S_{\mathrm{pert}}^{(0,1)}\left[F_{\infty}\left[\mathcal{L}_{H}+\mathcal{L}_{L}\right]\right]-\int F_{M}^{(0,1)}\left[\mathcal{L}_{L}\right] \\
& =\int\left(F_{\infty}^{(0,1)}\left[\mathcal{L}_{L}\right]-F_{M}^{(0,1)}\left[\mathcal{L}_{L}\right]\right)
\end{aligned}
$$

On the right hand side occur all the instanton-light particle interactions, and there is a cancellation for all instantons with $\mu_{I}<M$. That is, $\int \delta \mathcal{L}^{(0,1)}$ is the sum over (the low energy approximation to) all the instanton - light particle interactions, with the instanton scales all running from $M$ to $\infty$ (see fig. 10). 


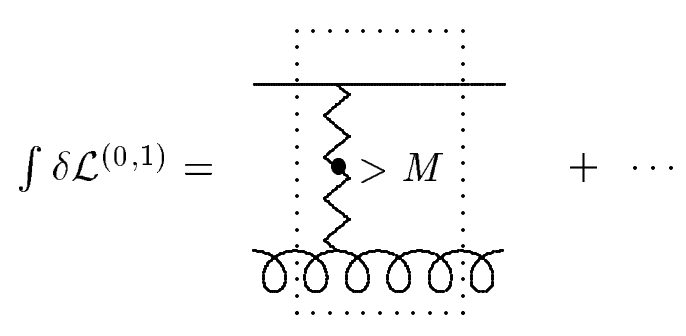

Figure 10: Matching contribution at $(0,1)$.

For each instanton diagram, there is

a corresponding anti-instanton diagram.

\subsection{QCD Calculation to order $(1,1)$}

To this order equation (4.9) gives

$$
\begin{aligned}
\delta \mathcal{L}^{(1,1)}=S_{\mathrm{pert}}^{(1,1)} & {\left[F_{\infty}\left[\mathcal{L}_{H}+\mathcal{L}_{L}\right]\right]-S_{\mathrm{pert}}^{(1,1)}\left[F_{M}^{[0,1]}\left[\mathcal{L}_{L}\right]+\delta \mathcal{L}^{[0,1)}\right] } \\
& -\int F_{M}^{(1,1)}\left[\mathcal{L}_{L}+\delta \mathcal{L}^{[1,0]}\right] \\
=S_{\mathrm{pert}}^{(1,1)} & {\left[\mathcal{L}_{H}+\mathcal{L}_{L}+F_{\infty}^{(0,1)}\left[\mathcal{L}_{H}+\mathcal{L}_{L}\right]\right] } \\
& -S_{\mathrm{pert}}^{(1,1)}\left[\mathcal{L}_{L}+F_{M}^{(0,1)}\left[\mathcal{L}_{L}\right]+\delta \mathcal{L}^{(0,1)}\right] \\
& -\int F_{M}^{(1,1)}\left[\mathcal{L}_{L}+\delta \mathcal{L}^{(1,0)}\right]
\end{aligned}
$$

This seems to express the matching information at this order in its most impenetrable form, so we will examine the right hand side term by term. Though we will discuss examples of only the instanton diagrams, the anti-instanton diagrams are present as well. Term $(5.10 a)$ is the sum over all diagrams with either a heavy or light particle loop, and with an instanton running over all scales (see fig. 11). Term (5.10b) is the sum over all diagrams with a light particle loop and either with an instanton whose scale runs from 0 to $M$, or with the low energy approximation to an instanton interaction where the instanton scale runs from $M$ to $\infty$ (see fig. 12). Finally, term (5.10c) is the sum over diagrams with (the low energy approximation to) a heavy fermion loop and an instanton whose scale ranges from 0 to $M$ (see fig. 13). 


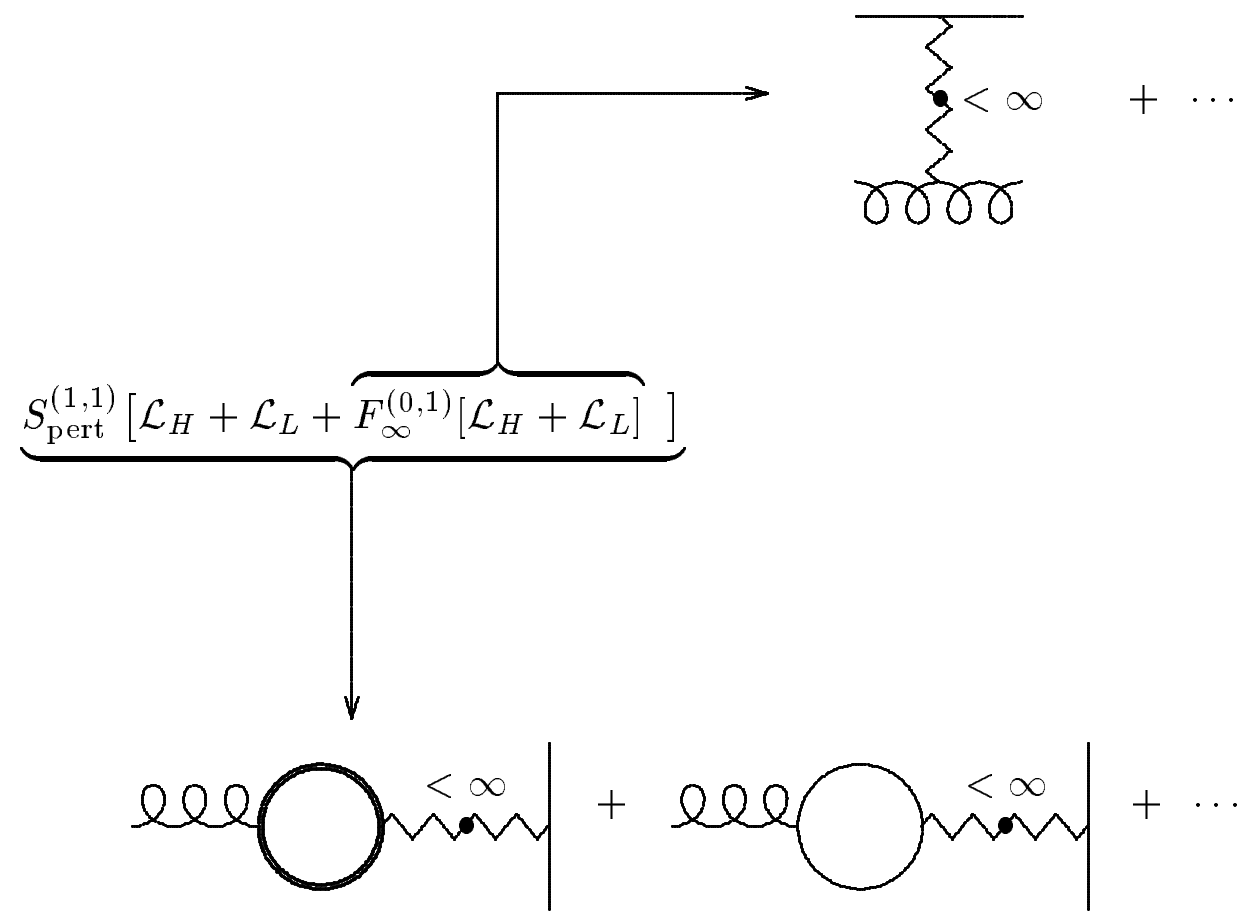

Figure 11: Example diagrams for term $(5.10 a)$.

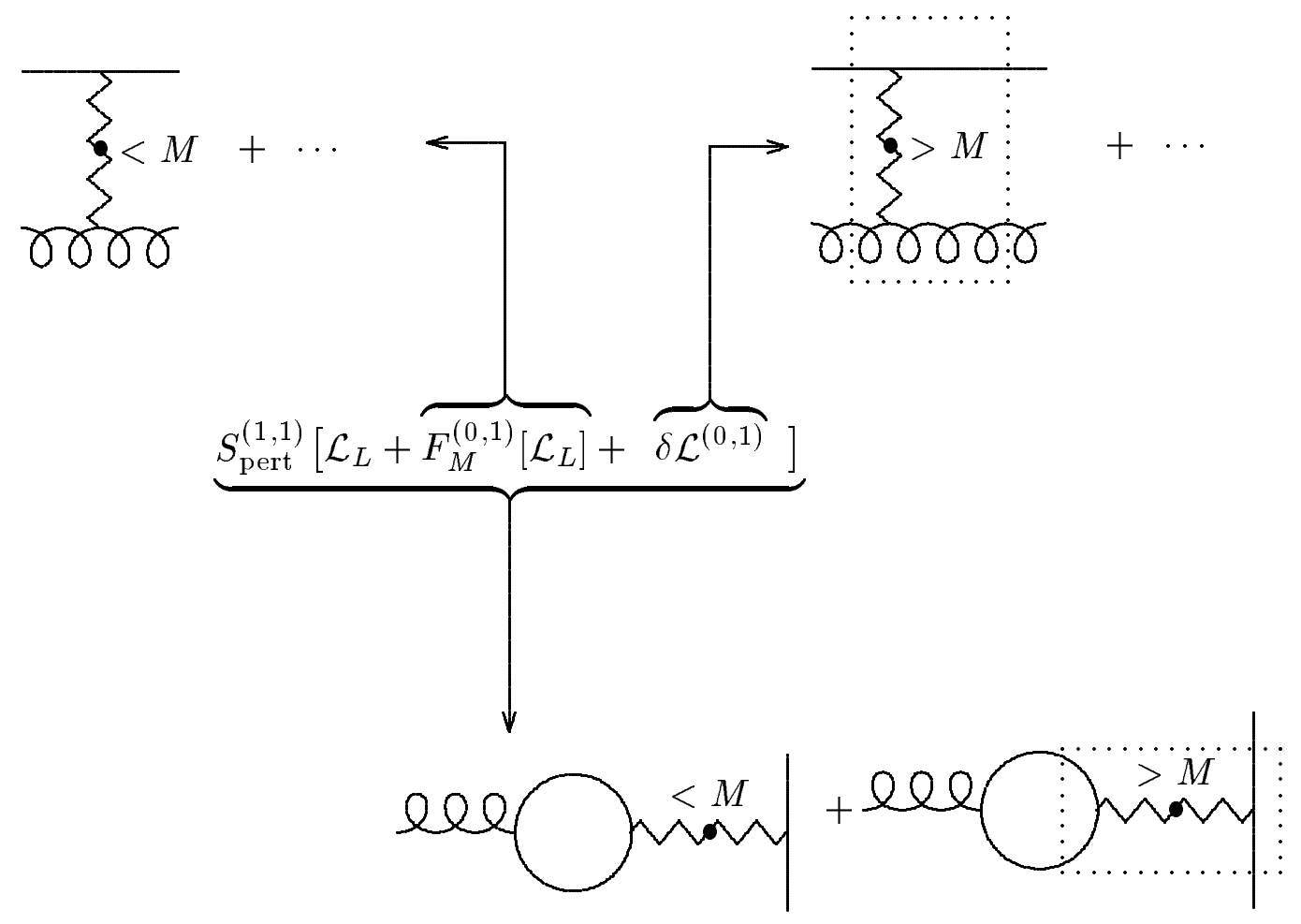

Figure 12: Example diagrams for term (5.10b). 


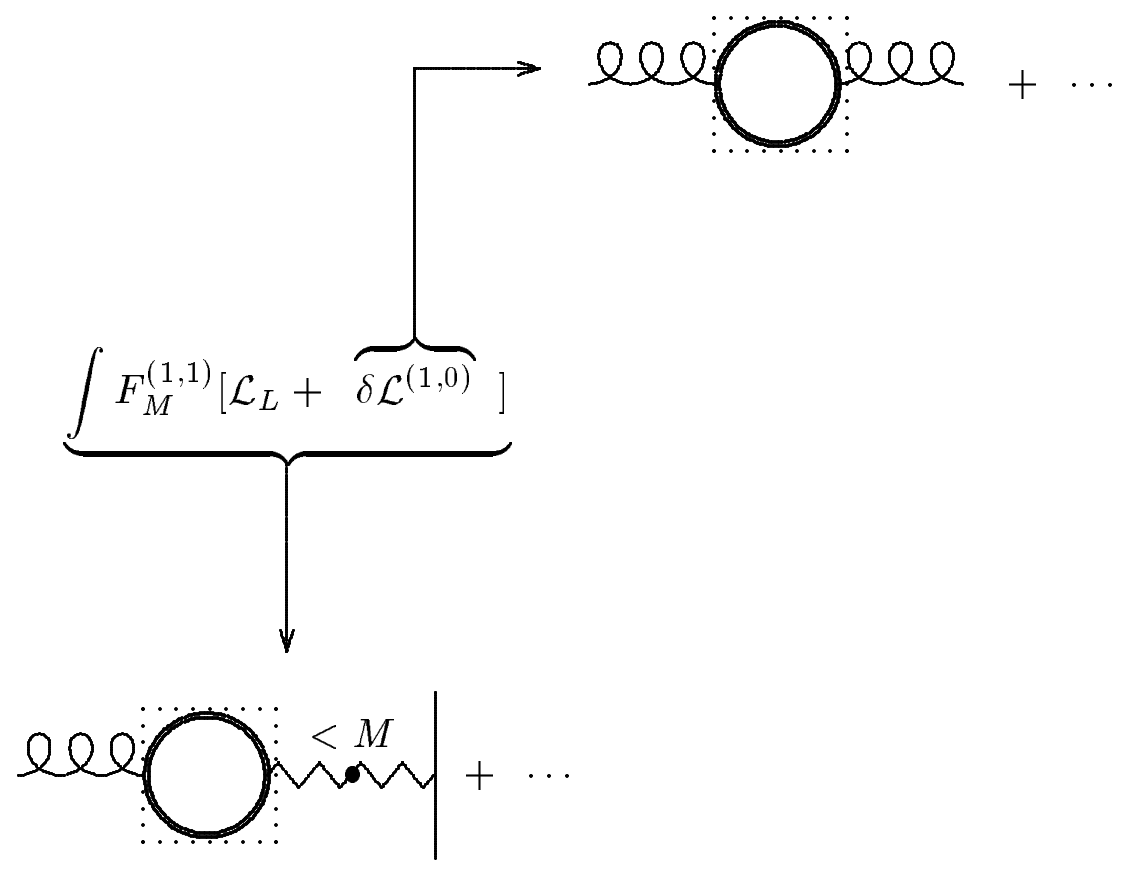

Figure 13: Example diagrams for term $(5.10 c)$.

Then, for $\int \delta \mathcal{L}^{(1,1)}$, equation (5.10) gives the diagrams indicated in fig. 14 .

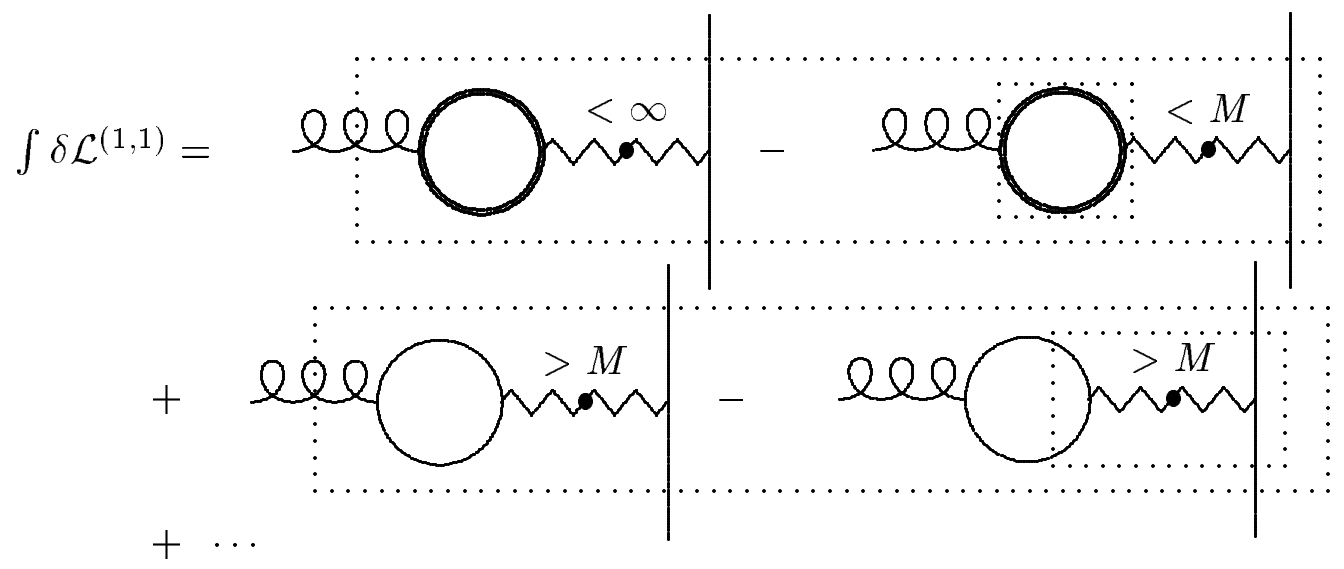

Figure 14: Matching contribution at $(1,1)$.

For each instanton diagram, there is a corresponding anti-instanton diagram.

The grouping of terms in fig. 14 is meant to be suggestive, in that low energy approximation boxes have been drawn around pairs of diagrams whose IR physics cancels within each pair. It should be stressed that a low energy box merely indicates the finite order momentum expansion through which $\delta \mathcal{L}^{(l, d)}$ is defined, so that the same result for $\delta \mathcal{L}^{(l, d)}$ is indicated whether a single box is drawn around the complete set of diagrams contributing 
to $\delta \mathcal{L}^{(l, d)}$ or several are drawn, one around each element in any allowed partition of the set of diagrams. A partition is only allowed, however, if each element is IR analytic, otherwise the momentum expansion of that element fails. Fig. 14 is meant to suggest a partition of the set of diagrams for which each element consists of the fewest allowable number of diagrams. In the first low energy box in fig. 14, the first term represents diagrams consisting of a heavy fermion loop with an instanton running over all scales. From these are subtracted diagrams represented by the second term: each is nominally the same diagram as one indicated in the first term, except the heavy fermion loop has been replaced by its low energy approximation, and the instanton scales run only from 0 to $M$. To this must be added the diagrams represented those in the second low energy box: the first term represents all diagrams consisting of a light fermion loop and an instanton whose scale runs from $M$ to $\infty$. From this must be subtracted the diagrams represented by the second term: each is nominally the same diagram as one in the first term, except that the instanton-background propagators have been replaced by their low energy approximation.

We have seen that in all the terms of $\delta \mathcal{L}$ up to $\mathcal{O}(1,1)$, the essential features of the second section have continued to hold. All infrared divergences, including those coming from large instantons, cancel out of the matching calculation. This is most interesting in the expression for $\delta \mathcal{L}^{(1,1)}$. For example in the first low energy box in fig. 14, the large instantons cancel because the larger the instanton, the better the approximation to the heavy fermion loop in the second term. It should also be stressed that, for example, the first and second terms of the second low energy box do not cancel exactly when the momentum expansion is carried out only to some finite order.

\section{Practical Calculations}

For a given term induced by instantons in the low energy theory, there are only a finite number of diagrams to calculate at each order in $(l, d)$. It is unlikely that all of these terms could be calculated analytically, however, because some of them involve the propagation of massive particles in an instanton background.

In nonabelian gauge theory, such a propagator for a massless particle of arbitrary spin and isospin can be calculated in closed form [13]. Also, the corresponding massive

propagator has been related to that of a scalar [14]. However, we believe the scalar massive propagator has not been calculated in closed form. 
In addition, the requirement that the quantum fluctuations about the instanton in the zero-mode direction be treated specially forced us to non-trivially modify the propagator of the field in which the instanton is found (see equation (3.16)). However, it is quite possible that this new propagator could still be calculated in closed form using methods similar to [13].

Whatever difficulties there are in exact analytic calculations, these diagrams could perhaps be calculated numerically or estimated analytically in certain regimes.

Finally, it seems likely that, in many calculations, instanton effects in the low energy theory will dominate any of the instanton induced matching corrections described here. This is especially true in a classically scale invariant (in the bosonic sector), asymptotically free theory like QCD, where infrared dominance seems to be a feature of most instanton calculations. We feel that it is nonetheless useful to have a method for systematically calculating the corrections, both for conceptual reasons and because they may sometimes be important.

\section{Acknowledgements}

STO wishes to thank Steve Hsu and Shane Hughes for useful discussions. This work was supported in part by the National Science Foundation, under grant \#PHY9218167, and in part by the Texas National Research Laboratory Commission under grant \#RGFY93-278B. The original idea for this work grew out of discussions between HG and Guido Martinelli and others at the Institute for Theoretical Physics in Santa Barbara. HG is grateful to the staff of the ITP for its hospitality and help. 


\section{References}

[1] H. Georgi, HUTP-93/A003, to appear in Ann. Rev. Nucl. Part. Sci., 43.

[2] T. Appelquist and J. Carazone, Phys. Rev. D11 (1975) 2856.

[3] Stephen D.H. Hsu, HUTP-93.

[4] R. Rajaraman, Solitons and Instantons, (Elsevier Science Publishers, Amsterdam, 1987).

[5] A.M. Polyakov, Nuc. Phys. B120 (1977) 429.

[6] I. Affleck, Nuc. Phys. B191 (1981) 429.

[7] G. 't Hooft, Phys. Rev. D14 (3432) 1976.

[8] I.I. Balitsky and A.V. Yung, Phys. Lett. B168 (1986) 113.

[9] See, e.g., S. Pokorski, Gauge Field Theories, (Cambridge University Press, Cambridge, 1989).

[10] S. Coleman, Aspects of Symmetry, (Cambridge University Press, Cambridge, 1988).

[11] See, e.g., N. Andrei and D.J. Gross, Phys. Rev. D18 (1978) 468.

[12] L.F. Abbott, Nuc. Phys. B185 (1981) 189

[13] L.S. Brown, R.D. Carlitz, D.B. Creamer, and C. Lee, Phys. Lett. 70B (1977) 180; Phys. Lett. 71B (1977) 103; Phys. Rev. D17 (1978) 1583.

[14] L.S. Brown and C. Lee, Phys. Rev. D18 (1978) 2180. 